\title{
BMJ Open Developing and validating an instrument to assess non-hospital health centers' preparedness to provide initial emergency care: a study protocol
}

\author{
Mehrdad Amir Behghadami, ${ }^{\oplus 1,2}$ Ali Janati, ${ }^{1}$ Homayoun Sadeghi-Bazargani, ${ }^{3}$ \\ Masoumeh Gholizadeh, ${ }^{1}$ Farzad Rahmani, ${ }^{4}$ Morteza Arab-Zozani ${ }^{\odot}$
}

To cite: Amir Behghadami M, Janati A, Sadeghi-Bazargani $\mathrm{H}$, et al. Developing and validating an instrument to assess non-hospital health centers' preparedness to provide initial emergency care: a study protocol. BMJ Open 2019;9:e026651. doi:10.1136/ bmjopen-2018-026651

- Pre-publication history and additional material is published online only. To view please visit the journal online (http://dx.doi. org/10.1136/bmjopen-2018026651).

$\mathrm{MAB}$ and $\mathrm{AJ}$ contributed equally.

Received 14 September 2018 Revised 23 June 2019 Accepted 28 June 2019

A Check for updates

(c) Author(s) (or their employer(s)) 2019. Re-use permitted under CC BY-NC. No commercial re-use. See rights and permissions. Published by BMJ.

For numbered affiliations see end of article.

Correspondence to Professor Ali Janati; janati1382@gmail.com, behghadami.m@gmail.com

\section{ABSTRACT}

Introduction Basic emergency management in urban and rural areas is a critical challenge. This paper presents the protocol for developing, validating and piloting an instrument to assess the preparedness of non-hospital health centres which deliver initial emergency care. Methods and analysis This study will be designed based on a sequential exploratory mixed method in two phases, in each of which there are three steps. In the first step of phase I, an extensive systematic review will be conducted. In the second step, through focus group discussions (FGDs), the experts' views on the definition of domains and items of the instrument will be compiled. In addition, semistructured interviews with the target group will be performed. Then, qualitative framework analysis will be performed. In the last step of phase I, the results of both steps will be triangulated by a panel of experts to define the domains and items to be included in the instrument. Phase II will include content validity, feasibility and reliability of the instrument. Content validity of the instrument will be assessed using modified Kappa coefficient based on clarity and relevance criteria. Feasibility of the instrument will be assessed through its implementation in 10 non-hospital health centres in Tabriz, which will be selected randomly. Reliability will be assessed in a pilot on 30 non-hospital health centres through using stratified random sampling method. Reliability of the instrument will be assessed through measuring internal consistency, test-retest reliability and inter-rater agreement. The main statistical methods for assessing reliability will include Cronbach's alpha, intraclass correlation coefficient and Kendall's tau-b. All the statistical analyses will be performed using Stata V.14. Ethics and dissemination This study is approved by Tabriz University of Medical Sciences Research Ethics Committee (IR.TBZMED.REC.1397.145). The results of the study will be presented at national scientific conferences and published in peer-reviewed journals.

\section{INTRODUCTION}

Under any condition, ranging from delays in the arrival of ambulances to critical extraordinary situations, ${ }^{12}$ non-hospital health centres, as social organisations and the most significant

\section{Strengths and limitations of this study}

- To our knowledge, this is the first protocol focusing on developing and validating an instrument to assess non-hospital health centre preparedness to provide initial emergency care in Iranian healthcare system.

- A potential strength of the study might be the variety of methods, that is, systematic review and quantity and quality study, in the development and validation of this instrument.

- All stages of the systematic review namely screening, quality appraisal, data extraction will be independently done by two researchers.

- In order to assess reliability of the instrument, two intrarater and inter-rater reliability assessment methods will be used.

- Due to limited resources, it is not possible to conduct this study on a larger scale.

centres of healthcare system, ${ }^{3}$ ought to provide basic life support through employing organised infrastructures such as physical space, medical equipment, emergency medicines, support facilities and human forces. This includes primary healthcare centres (PHCC) and outpatient clinics, which are the mainstay of healthcare throughout many of the rural and urban areas of low-income countries. ${ }^{4}$

Non-hospital centres are in charge of delivering integrated care services to people in defined areas ${ }^{5}$. Such centres are mainly staffed by clinical officers, nurses and physicians. In Iran, the duties assigned to these providers include prevention of illness, minor surgery and initial emergency care for the life-threatening emergencies. For this reason, providers must be prepared to deal with emergency situations that may occur in the geographic area of activity of these centres. Also, they must collaborate with emergency medical services (EMS). ${ }^{6}$ 
Along with hospitals, specialty clinics and polyclinics, participation of non-hospital health centres in providing emergency care is an advantage which provides the highest number of possible services to the highest number of potential individuals. ${ }^{7}$ In many cases, such non-hospital health centres represent the first access for the injured to healthcare system. ${ }^{4}$ In addition, since life-threatening hazards always occur accidentally and unexpectedly, ${ }^{7}$ it is expected that non-hospital health centres be prepared to provide patients with chronic and acute cases of disease with a range of emergency care. ${ }^{8}$

At least half of the patients with acute cardiovascular diseases suffer angina pectoris as well. Indeed, one-fourth of these patients have been diagnosed to have had a heart attack. ${ }^{9}$ If such patients get inflicted with cardiac arrest, family physicians (FPs), particularly in rural areas, often are the first healthcare professionals to commence cardiorespiratory resuscitation. Hence, so as to improve acute myocardial infarction survival and decrease mortality rate of such patients, PHCC providers should be ready to provide emergency primary cares. ${ }^{10}$ It is undeniable that out-of-hospital cardiac arrest survival is still poor; however, it has been observed that the FPs who have witnessed cardiac arrest and performed emergency defibrillation have been successful in referring about half of the patients with cardiac arrest to hospitals. The range of their success is $12.5 \%$. Moreover, $11.6 \%$ of serious emergency cases, which require resuscitation, are performed by FPs. ${ }^{11}$

Providing emergency care at non-hospital health centre level as a means of reducing hospitals' emergency ward load has been recommended by a variety of studies. ${ }^{6} 7$ 12-20 Hence, it is expected that basic life support be provided in cases of emergency. ${ }^{11}$ Nevertheless, the question is if non-hospital health centres have been appropriately organised to offer emergency care or not. Most of the international studies consistently have reported the unpreparedness of non-hospital health centres for offering emergency care in terms of emergency medicine, equipment and support facilities. ${ }^{10} 11$ 21-25 Therefore, preparedness of non-hospital health centres can play a pivotal role in offering desirable emergency care to patients. ${ }^{26}$

A review of the published literature highlights a dearth of studies on this subject. Although majority of the studies have focused on assessing emergency care offered in hospitals, there is a published international study which has investigated the emergency care in PHCC. This study (2007) was carried out in Abha, Southwest Saudi Arabia. It applied Donabedian triple model (structure, process and outcomes) to assess the quality of emergency care in PHCC. ${ }^{7}$ There is only one national study on assessing non-hospital health centres status for providing emergency care. Generally, they have reported deficiencies in infrastructures and support facilities for offering emergency care through using checklists (general characteristics of the centres and views of the authorities and health staff toward offering urgent care, equipment and necessary and basic medicine) ${ }^{6}$
Based on the investigation of the researchers, there is no study done on developing an instrument to assess preparedness of non-hospital health centres providing initial emergency care. Hence, in accordance with healthcare system in Iran, a valid and reliable instrument will be developed to assess preparedness of non-hospital health centres including rural and urban community healthcare centres, and outpatient clinics for basic emergency management (basic support). The main aim of the study is to develop a valid and reliable instrument to measure preparedness of non-hospital health centres to provide emergency care.

\section{Research objectives}

The objectives of this protocol will be as follow' ${ }^{1}$ identifying domains and items of the instrument to assess preparedness of non-hospital health centres to provide initial emergency care through using a systematic review, ${ }^{2}$ exploring domains and items associated with assessing preparedness from the perspective of experts and target groups and, finally, ${ }^{3}$ assessing validity and reliability of the instrument.

\section{METHODS AND ANALYSIS}

\section{Study design}

The protocol of the study will be designed based on a sequential exploratory mixed-method in two phases, in each of which there are three steps (figure 1). Sequential exploratory mixed-method is applied when a researcher does not know the most important concepts for the study; plus, when there is no suitable instrument for measuring the intended concept. Sequential exploratory mixed method includes both qualitative and quantitative

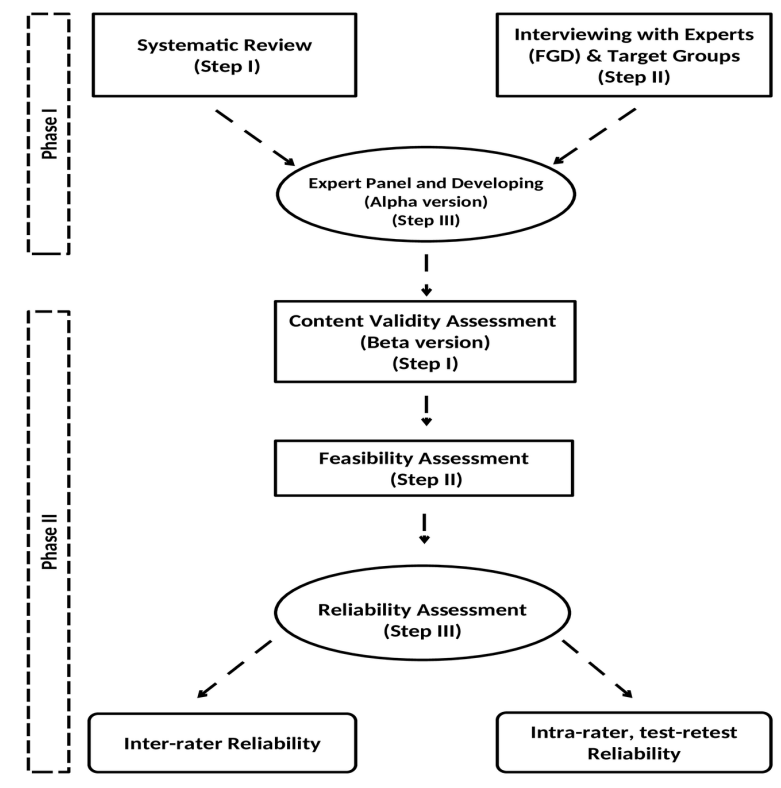

Figure 1 Phases and steps of each phase of developing an instrument. 
methods where results of the qualitative method can help develop the quantitative method. ${ }^{27}$

\section{Phase I: instrument development}

This phase will be conducted in three steps, including systematic review, FGDs with experts and semistructured interviews with the target group (emergency care providers and recipients), and a panel of experts. The planned time frame for phase I is 13 months (April 2018April 2019).

\section{Phase I: step I}

In the first step, a systematic review will be conducted to identify the domains and items related to assessing preparedness of non-hospital health centres, which offer initial emergency care. We will conduct a systematic review in accordance with the Preferred Reporting Items for Systematic Reviews (PRISMA). We will use the PRISMA because its use would reduce the risk of flawed reporting and increase clarity of the systematic review reports. ${ }^{28}$

The review protocol has already been registered in PROSPERO (registration no. CRD42018096044; http:// www.crd.york.ac.uk/PROSPERO/display_record.php? ID=CRD42018096044).

The proposed systematic review question is:

What are the domains and items related to assessing preparedness of non-hospital health centers to offer initial emergency care?

\section{Data sources and search strategy}

An extensive search of the literature will be conducted in five databases including PubMed, Scopus, Web of Science, Barakat Knowledge Network Systems (BKNS) and Scientific Information Database (SID) using a search strategy to identify the studies on non-hospital health centres' preparedness to provide initial emergency care. All of the searches will be done for the articles published in English and/or Persian without time limit up to March 2018.

SID is a database which categorises Iranian journals of science and research into different science groups. The database started its mission in 2004 (http://www.sid.ir/ En/Journal/).

Moreover, Barakat Knowledge Network System (BKNS) started its mission in seven science fields in 2012. Health was the first field to be administered by the system. Through providing a comprehensive database of the articles taken from health journals and conferences, this part of the system has turned Iran into information corridor of Islamic world (http://health.barakatkns.com) ${ }^{29}$. Indeed, we will use a combination of Medical Subject Headings (Mesh) terms and free terms to maximise sensitivity of the search. English key words and their Persian equivalents will be used. The key words include:

Health Center, Primary Care Center, Primary Health Care Center, Health Post, Health House, Rural Health Center, Urban Health Center, Health Care Center, Outpatient Clinic, Ambulatory Health Center,
Non-hospital, Emergency, Emergency Services, Emergency Care, Emergency Medical Services, Emergency Case, Critical Emergency, Basic Life Support, Basic Emergency Care, Basic Emergency Services, Primary Emergency Care, Primary Emergency Services, Trauma, Pre hospital, Preparedness, Ready, Readiness, Assess, Evaluation, Appraisal, Performance Evaluation, Performance Appraisal, Quality Assessment, Inspection.

The proposed search strategy for PubMed database is in online supplement file 1. Grey literature from governmental institutions or organisations (for instance, health ministry, treatment and health vice chancellery, East Azerbaijan Province) and other organisations (like WHO), will be used to identify other related studies including reports and etc. Additionally, the references lists and citation of the included studies will be hand-searched to identify other potentially related studies, which have not been recognised in the databases search. In order to cover the grey literature, 10 pages on Google Scholar will be searched.

\section{Study selection}

All of the records from the search will be imported into EndNote software package (V.X6); and duplicates will be discarded. Then, all the titles and abstracts and full text will be independently screened by two researchers based on the inclusion criteria. Any disagreements will be resolved through a dialogue between the two researchers. In cases where there will be no consensus or any question remains, a third researcher will be consulted.

\section{Eligibility criteria}

Inclusion and exclusion criteria for reviewing the title, abstract and full text will be as follows.

\section{Inclusion criteria}

Studies related to PHCC and outpatient clinics will be included. Studies, which report at least one domain and item related to preparedness, will be included as well.

\section{Exclusion criteria}

Studies conducted in emergency wards of hospitals, outpatient specialised clinics affiliated with hospitals and EMSs (115 Emergency) will be excluded from the study since offering emergency care is one of their main responsibilities. Hence, they should be completely prepared to deal with emergent cases.

The articles, whose full text is not available, along with letters to the editor and editorials will be excluded from the study. Studies associated with preparedness in human and natural disasters such as flooding, earthquakes, battles, etc will be excluded from the study as well. Also, the studies with low quality will be excluded.

\section{Quality assessment}

The included studies will be independently appraised by two researchers. And if necessary, potential discrepancies will be resolved through discussion between the two researchers and a third researcher. 
Methodology quality of all the included studies in the review will be assessed with a Mixed Methods Appraisal Tool (see online supplementary file 2 ). Validity and reliability of the tool have been approved, ${ }^{3031}$ and at present, MMAT is the best and most comprehensive tool available for assessing studies of multiple method types. MMAT is used to investigate all the three studies of methodological quality, including mixed methods, qualitative and quantitative (randomised controlled, non-randomised and descriptive). Total score of the methodology will be calculated as percentage. The criteria used to determine quality score are different from designing the study. Reliability of the tool has been tested before and has met the accepted standards. ${ }^{32}$

\section{Data extraction}

A data extraction form will be designed by the researchers. Primary researcher will independently extract data related to the study characteristics (eg, objectives of the study, type of the study, etc) from each included study. This will be checked by a second researcher for accuracy. Main characteristics of the included studies and their corresponding results will be summarised based on the following data: the domains assessed to indicate the non-hospital health centres preparedness to provide initial emergency care, type of the centre, publication year of the studies, language of the study, type of the study (qualitative, quantitative, mixed or other) and MMAT score including score $0 \%$ (no quality), 25\% (low quality), $50 \%$ (moderate quality), $75 \%$ (considerable quality) and $100 \%$ (high quality).

Data extraction and the coding related to content items will be independently conducted by two researchers with a random sample. Then, the included studies will be assessed for consistency to show their consistency in the researchers' views.

\section{Data synthesis}

Data will be synthesised through using directed content analysis method ${ }^{3031}$ and will be categorised based on Donabedian model. ${ }^{32}$

This involves coding of the items identified from studies into domains and sub-domains. The process will be conducted by using deductive reasoning. ${ }^{31}$ Directed content analysis is guided by a structured approach, which uses existing theory (or research) to identify the key concepts as initial coding categories. ${ }^{31}$ Our proposed initial coding categories will be identified from previously published regulatory guidance and existing research on non-hospital health centres' preparedness to provide initial emergency care. Data collection and analysis will pursue an iterative process, whereby the coding structure and emerging domains will be continuously extended and refined. The primary researcher will lead the qualitative data collection and analysis process, and intermittently consult with the secondary researcher on the coding structure and emergent domains. Any disagreements will be resolved through consultation with a third researcher, if necessary.

\section{Phase I: step II}

Study design, data collection and participants

In the second step of developing the instrument, proportionate to health services providing system in Iran and based on emergency care (basic life support), FGDs meetings, presumably at least 3 meetings with $8-10$ persons in each, and semistructured interviews are predicted. Those who fail to participate in FGDs will have a semistructured interview.

Prior to holding FGDs, exact time of the sessions will be arranged with the participants via e-mail or telephone. Then, the invitation letter, including introduction of the study, its aims and also the time and place of the session, will be sent to the participants through administrative automation of Tabriz University of Medical Sciences. Additionally, 1 day before the meeting, the participants will be notified of the time and location of the meeting. The FGDs with the experts will be held in the conference room of the health service management department. Each FGD meeting will be planned for 2-3 hours, including the breaks with refreshments. On condition that no information is obtained from the FGDs or differences are found among the FGDs, additional FGDs will be performed until saturation has been achieved. Discussions will be conducted by two skilled or trained moderators and one assistant.

The people participating in the FGD meetings include specialists in emergency medicine, PHC and executives of health centres, with over 5 years of work experience, and EMS experts. Purposive sampling method will be used to identify those who have sufficient knowledge in our research scope. Based on the objectives of the study and also in order to benefit more from the samples, the researchers will select individuals who can respond to the study questions. This type of sampling can be useful when it is needed to reach the targeted sample rapidly, and where sampling for proportionality is not the main concern. Regarding gender, age, occupation and education of specialists, sampling will be done with maximum variation.

In addition, semistructured interviews will be conducted through using a predetermined interview guide to increase quality of instrument questions with the target group (emergency care providers and recipients). Semistructured interviews will be conducted at non-hospital centres in Tabriz, northwest of Iran. A series of semistructured interviews will be conducted among a purposive sample of physicians and nurses who deliver initial emergency care to patients. Sampling will be done until theoretical saturation is reached (estimated to be 12 participants). Semistructured interviews will be conducted at the participants' work place. Moreover, a series of semistructured interviews will be conducted with a convenience sample of recipients of these non-hospital centres. Sampling will be done until theoretical saturation is reached (estimated 
to be 10 participants). The purpose of these interviews is to explore the factors affecting initial emergency care provided in non-hospital health centres from the perspective of these individuals.

Before conducting any semistructured interview and FGD, the equipment required for recording and interviewing will be supplied. All the semistructured interviews and FGDs will be transcribed and immediately recorded word by word. They will be considered by two researchers, one of whom has extensive experience in this field, so as to preserve validity and reliability. The semistructured interviews will go on up to the time when the data are saturated and there is no new information concerning the phenomenon left. Moreover, in order to have consistent and accurate data, through participants' validity method, after every interview, the target group will be asked to approve the accuracy of the presented points.

\section{Data analysis}

A framework analysis approach will be employed to manually analyse the interview transcripts from all the FGDs and semistructured interviews. According to the framework analysis, data analysis will be carried out after a fivestage process, consisting of familiarisation, identifying a thematic framework, indexing, charting and mapping and interpretation. ${ }^{33}$ Each stage of the analysis will be performed by the first researcher. In order to improve inter-rater reliability and prevent data bias, a selection of transcripts will be reviewed by another researcher.

The Donabedian model will be used as a theoretical framework to categorise the synthesised domains. This model emphasises the importance of structure, process and outcome. ${ }^{32}$

\section{Patient and public involvement}

Objectives, expected benefits of the study and type of the collected information will be explained to the participants. An informed consent will be obtained from every participant before the interview. Confidentiality of data will be ensured and maintained throughout the whole study. Specialists and emergency care providers and recipients will engage in data collection process. Semistructured interviews will be carried out to improve quality of the instrument questions with the recipients. Existence of a preparedness assessment instrument can play a pivotal role in identifying the capabilities of non-hospital health centres and offering desirable emergency care to patients. The instrument will be supplied in accordance with the needs of vice chancellery for health and lack of an instrument to assess preparedness of non-hospital centres in East Azerbaijan Province. This instrument will be used to assess all of the centres in the same way whereby it will be plausible to compare them in this regard. The results obtained from this study will lead to developing a valid and reliable tool, which will be employed by vice chancellery for health to monitor and assess the centres in East Azerbaijan Province.

\section{Phase I: step III}

In the third step, the domains and items of the respective instrument, which are extracted through using a systematic review, FGDs and interviews with the target group, will be examined by the research team in terms of overlapping and duplication. Then, in order to determine the ultimate domains and items, the table of specifications methodology will be used. ${ }^{34}$ A panel of people with expertise in the fields of emergency medicine will review the items in each domain. In practice, alignment of a category of items, which will be placed in the rows, with the constituting domains, which will be placed in the columns, will be considered. Next, the evidence will be quantitatively, based on the percentage of the specialists' consensus on the rows and columns of the table of specifications, and qualitatively, based on the specialists' feedback on what other items are added, compiled and analysed by the panel of experts. Finally, alpha version of the instrument will be designed.

\section{Phase II: content validity, feasibility and reliability testing of the proposed instrument}

The planned time frame for phase II is 5 months (May 2019-September 2019).

\section{Phase II: step I}

The first step of Phase II, will consist of content validity of the instrument that will be assessed through using modified Kappa coefficient (modified CVI). The aim of the assessment is to answer the question if content of the instrument will be capable to measure the defined objectives or not. In this regard, alpha version of the instrument will be sent to 10 selected specialists. The fields of specialty include: emergency medicine, PHC specialists and general practitioners working in clinics and PHCG and epidemiologists. The specialists will be asked to assess their own ideas to improve quality of the instrument and also judge the available items in terms of their clarity and relevance. Finally, the specialists will be asked to put forth any other question or recommendation they have to improve quality of the questions. Beta version of the instrument will be provided.

In order to validate the instrument, based on views of the specialists, CVI and modified KAPA (modified CVI) will be assessed. Moreover, to consider its content validity index, clarity and relevance, as two criteria, will be separately considered on a four-point Likert scale by the specialists. The modified Kappa coefficient and CVI will be calculated for the instrument and items. ${ }^{3536}$

To calculate modified kappa statistic, the probability of chance agreement will be calculated for each item by the following formula:

$$
P_{C}=[N ! / A !(N-A) !]^{*} \cdot 5^{N}
$$

In this formula, $\mathrm{N}$ stands for the number of experts and, A stands for the number of agreeing specialists.

After calculating I-CVI for all the instrument items, finally, kappa will be computed by entering the numerical 
values of probability of chance agreement $\left(\mathrm{P}_{\mathrm{C}}\right)$ and content validity index of each item (I-CVI) in the following formula:

$$
K=\left(I-C V I-P_{C}\right) /\left(1-P_{C}\right)
$$

Evaluation criteria for kappa, using guidelines described in Cicchetti and Sparrow ${ }^{37}$ and Fleiss (1981) is: Excellent $=\mathrm{k}>0.74$; Good=k of. 60-.74; and Fair=k of. 40 to. $59 .^{37}$

Polit states that after controlling items by calculating modified kappa, each item with I-CVI equal or higher than 0.78 would be considered excellent. Researchers should note that, as the number of experts in the panel increases, the probability of chance agreement decreases and values of I-CVI and kappa converge. ${ }^{35}$

\section{Phase II: step II}

In the second step, feasibility of the instrument concerning the simplicity of answering questions of the instrument and other potential problems in 10 non-hospital health centres in Tabriz will be assessed. Then, the required modifications will be made. For this aim, simple random sampling will be used.

\section{Phase II: step III}

In the last step of phase II, in order to assess reliability of the instrument, two intrarater and inter-rater reliability assessment methods will be used.

- Intra-rater، test-retest :

Measurement stability will be assessed over time. So as to assess internal reliability of the developed instrument, preparedness of non-hospital health centres will be assessed by an assessor team. Also, in order to conclude internal reliability, then a time interval from the first assessment, the assessor team will again assess the same non-hospital health centres. Depending on the issue, test-retest period will be considered to be short or long.

- Inter-rater agreement

In order to conduct inter-rater reliability assessment, non-hospital health centres will be divided into two categories and will be independently assessed by two assessor teams. The obtained results will be compared with internal reliability assessment. In the research phase, the centres' assessment will be performed by a team consisting of a physician and health system expert.

\section{Sample size}

In order to carry out the pilot test, there are at least 30 non-hospital health centres available as sample of the study. However, considering variety of the centres and considering other related conditions, stratified random sampling will be employed. Sample framework for selecting strata will be based on the list obtained from health and treatment chancellery.

\section{Analysis}

All the statistical analyses will be performed through using Stata V.14 statistical software package. Reliability of the instrument will be assessed through internal consistency, as well as the test-retest and inter-rater reliability methods. Cronbach alpha will be used to measure the internal consistency for the total instrument and each item. For test-retest reliability of the instrument, the intra-class correlation coefficient in a time interval will be used to calculate it for the total instrument and each dimension. In addition to it, Kendal's Tau-b correlation coefficients will be reported for the reliability of test-retest.

\section{Ethics and dissemination}

Iran is a vast country with a great number of non-hospital health centres, which are in charge of promoting health in society. Part of this responsibility is prevention and part of it includes management of regional level emergency patients. It is necessary that any of these centres be assessed to ensure that the quality and standards of the cares are uniform.

In the present study, the developed instrument will help to assess preparedness rate of non-hospital health centres in response to emergency referrals, get rid of any deficiencies and motivate policy makers, executive managers and patients to make use of this potential capacity. The most positive point of the present study is that it will design the first comprehensive and systematic instrument in the field through resorting to quantitative and qualitative studies.

The study has been approved.

Informed consent will be obtained from all the participants. We will disseminate the results of the study through publication in international peer-reviewed journals and at national conferences.

\section{Author affiliations}

${ }^{1}$ Iranian Center of Excellence in Health Management, Department of Health Service Management, School of Management and Medical Informatics, Tabriz University of Medical Sciences, Tabriz, Iran

${ }^{2}$ Student Research Committee (SRC), Tabriz University of Medical Sciences, Tabriz, Iran

${ }^{3}$ Road Traffic Injury Research Center, Tabriz University of Medical Sciences, Tabriz, Iran

${ }^{4}$ Emergency Medicine Department, Sina Medical Research and Training Hospital, Tabriz University of Medical Sciences, Tabriz, Iran

Collaborators Mehrdad Amir Behghadami; Ali Janati, Homayoun SadeghiBazargani; Masoumeh Gholizadeh; Farzad Rahmani; Morteza Arab-Zozani

Contributors MAB, AJ and HSB initiated and conceived the study. MAB and AJ participated in the study design through consulting HSB, MG and FR. MAB drafted the research protocol. AJ, HSB and MA-Z critically revised the content of the written protocol. All the authors approved the final version of this protocol.

Funding This study was approved by Tabriz University of Medical Sciences. This work was supported by Tabriz University of Medical Sciences, Tabriz, Iran (Grant number: 59656).

\section{Competing interests None declared.}

Patient consent for publication Obtained.

Ethics approval This study was approved by Tabriz University of Medical Sciences (ethical confirmation number; IR.TBZMED.REC.1397.145).

Provenance and peer review Not commissioned; externally peer reviewed.

Author note This study protocol will be of MAB's MSc dissertation, supervised by $\mathrm{AJ}, \mathrm{MG}$, and $\mathrm{HSB}$.

Open access This is an open access article distributed in accordance with the Creative Commons Attribution Non Commercial (CC BY-NC 4.0) license, which permits others to distribute, remix, adapt, build upon this work non-commercially, 
and license their derivative works on different terms, provided the original work is properly cited, appropriate credit is given, any changes made indicated, and the use is non-commercial. See: http://creativecommons.org/licenses/by-nc/4.0/.

\section{REFERENCES}

1. Jaeger MW, Ambadwar PB, King AJ, et al. Emergency Care of Children with Ambulatory Care Sensitive Conditions in the United States. J Emerg Med 2015;49:729-39.

2. Malek ML, Haghpanah S, Moravej $\mathrm{H}$, et al. The effect of intervention on patient's satisfaction in emergency departments of the hospitals affiliated to shiraz university of medical sciences. 2010.

3. Montalto M. Outside the hospital: the delivery of healthcare in nonhospital settings. Public Health Assoc Australia Inc Po Box 319 Curtin, Act 2600, Australia 2009.

4. Mock C. Guidelines for essential trauma care: World Health Organization. 2004.

5. Tabrizi JS, Behghadami MA, Saadati M, et al. Self-care Ability of Older People Living in Urban Areas of Northwestern Iran. Iran J Public Health 2018;47:1899-905.

6. Heidari A, Kabir MJ, Jafari N, et al. Assessment of human and physical resources in health houses and health-care centers providing emergency services: a study in Golestan province. Journal of Health in the Field 2016;3(4).

7. Mahfouz AA, Abdelmoneim I, Khan MY, et al. Primary health care emergency services in Abha district of southwestern Saudi Arabia. East Mediterr Health J 2007;13(1):103-12.

8. de Almeida AO, Araújo IE, Dalri MC, et al. Theoretical knowledge of nurses working in non-hospital urgent and emergency care units concerning cardiopulmonary arrest and resuscitation. Rev Lat Am Enfermagem 2011;19:261-8.

9. Meal AG, Pringle M, Hammersley V. Time changes in new cases of ischaemic heart disease in general practice. Fam Pract 2000;17:394-400.

10. Johnston CL, Coulthard MG, Schluter PJ, et al. Medical emergencies in general practice in south-east Queensland: prevalence and practice preparedness. Med J Aust 2001;175:99-103.

11. Yorganci M, Yaman H. Preparedness of primary healthcare centers for critical emergency situations in southwest Turkey. Prehosp Disaster Med 2008;23(4):342-5.

12. Rehmani R, Norain A. Trends in emergency department utilization in a hospital in the Eastern region of Saudi Arabia. Saudi Med $J$ 2007;28:236-40.

13. Siddiqui S, Ogbeide DO. Utilization of emergency services in a community hospital. Saudi Med J 2002;23:69-72.

14. Selasawati HG, Naing L, Wan Aasim WA, Wan AW, et al. Inappropriate utilization of emergency department services in Universiti Sains Malaysia hospital. Med J Malaysia 2004;59:26-33.

15. Lowy A, Kohler B, Nicholl J. Attendance at accident and emergency departments: unnecessary or inappropriate? J Public Health 1994;16:134-40.

16. Khangura JK, Flodgren G, Perera R, et al. Primary care professionals providing non-urgent care in hospital emergency departments. The Cochrane Library 2012.

17. Furber AS. Referral to hospital in Nepal: 4 years' experience in one rural district. Trop Doct 2002;32:75-8.
18. Carret ML, Fassa AC, Domingues MR. Inappropriate use of emergency services: a systematic review of prevalence and associated factors. Cad Saude Publica 2009;25:7-28.

19. Bosch X, Escoda O, Nicolás D, et al. Primary care referrals of patients with potentially serious diseases to the emergency department or a quick diagnosis unit: a cross-sectional retrospective study. BMC Fam Pract 2014;15:75

20. Shah NM, Shah MA, Behbehani J, Jaafar B. Predictors of non-urgent utilization of hospital emergency services in Kuwait. Soc Sci Med 1996;42:1313-23.

21. Toback SL. Medical emergency preparedness in office practice. Am Fam Physician 2007;75(11):1679-84.

22. Fuchs S, Jaffe DM, Christoffel KK. Pediatric emergencies in office practices: prevalence and office preparedness. Pediatrics 1989;83:931-9.

23. Liddy C, Dreise H, Gaboury I. Frequency of in-office emergencies in primary care. Can Fam Physician 2009;55(10):1004-5.

24. Heath BW, Coffey JS, Malone P, et al. Pediatric office emergencies and emergency preparedness in a small rural state. Pediatrics 2000;106:1391-6.

25. Schweich PJ, DeAngelis C, Duggan AK. Preparedness of practicing pediatricians to manage emergencies. Pediatrics 1991;88:223-9.

26. Jahani M, Rastgar A, Hosseinipour M, et al. Structure of space, personnel and equipment of hospital emergency department of Babol university of medical sciences based on national standards. 2009.

27. Creswell JW, Clark VLP. Designing and conducting mixed methods research: Sage publications, 2017.

28. Liberati A, Altman DG, Tetzlaff J, et al. The PRISMA statement for reporting systematic reviews and meta-analyses of studies that evaluate health care interventions: explanation and elaboration. PLoS Med 2009;6:e1000100.

29. Amir Behghadami M, Janati A. A critical appraisal of the review study to improve its reporting quality. Iranian J Nursing Midwifery Res 2019;24:313.

30. Hsieh HF, Shannon SE. Three approaches to qualitative content analysis. Qual Health Res 2005;15:1277-88.

31. Potter WJ, Levine-Donnerstein D. Rethinking validity and reliability in content analysis. 1999.

32. Donabedian A. An introduction to quality assurance in health care: Oxford University Press, 2002.

33. Furber C. Framework analysis: a method for analysing qualitative data. Afr J Midwifery Womens Health 2010;4:97-100.

34. Newman I, Lim J, Pineda F. Content validity using a mixed methods approach: Its application and development through the use of a table of specifications methodology. Journal of Mixed Methods Research 2013;7:243-60.

35. Polit DF, Beck CT, Owen SV. Is the CVI an acceptable indicator of content validity? Appraisal and recommendations. Res Nurs Health 2007;30:459-67.

36. Haynes SN, Richard DCS, Kubany ES. Content validity in psychological assessment: A functional approach to concepts and methods. Psychol Assess 1995;7:238-47.

37. Cicchetti DV, Sparrow SA. Developing criteria for establishing interrater reliability of specific items: applications to assessment of adaptive behavior. Am J Ment Defic 1981;86:127-37. 